\author{
Review Article \\ www.ijrap.net
}

(ISSN Online:2229-3566, ISSN Print:2277-4343)

\title{
A CRITICAL REVIEW ON PUSHKARMOOLADI YOGA IN SHWASA ROGA
}

Khobragade Swapnil ${ }^{1}$, Upadhyay Prem Shanker ${ }^{2 *}$

${ }^{1}$ PG Scholar, Department Kaumarbhritya/Balroga, Faculty of Ayurveda, Institute of Medical Sciences, BHU,

Varanasi, U.P., India

${ }^{2}$ Associate Professor, Department of Kaumarbhritya/Balaroga, Faculty of Ayurveda, Institute of Medical Science, BHU Varanasi, U.P., India

Received on: 08/08/21 Accepted on: 15/11/21

*Corresponding author

E-mail: psupadhyay08@gmail.com

DOI: 10.7897/2277-4343.1206172

\begin{abstract}
Shwasa is a disease of Pranavaha strotas. It is clinically correlated with bronchial asthma. Symptoms of Shwasa are nearly similar to Bronchial Asthma, a chronic inflammatory disorder of the airways. Pushkarmoladi yoga is described in Yoga Ratnakara and Bhaishya Ratnavali. This yoga contains Pushkarmoola, Pippali, Dhanvayasa, Karkatshringi and Ativisha. This study was conducted to know the efficacy of Pushkarmoola diyoga in Shwasa (Bronchial Asthma). The literary review was performed by a collection of material related to the clinical efficacy of Pushkarmooladiyoga with the help of several essential Ayurvedic and Modern textbooks, Research papers and journals. Pushkarmooladi yoga shows bronchodilator property by relaxation of bronchial smooth muscles and antimicrobial activity against various gram +ve and gram-ve bacteria, which are responsible for Shwasa and have antioxidant properties due to the presence of the particular phenolic compound in its ingredients.
\end{abstract}

Keywords: Shwasa, Bronchial Asthma, Pranavaha Srotodushti, Antimicrobial activity.

\section{INTRODUCTION}

Bronchial asthma is an inflammatory disease of the airway. It leads to recurrent episodes of wheezing, breathlessness, chest tightness, and cough, particularly at night or early morning. Asthma is a non-communicable disease with significant public health consequences in children and adults, including high morbidity and mortality in severe cases. ${ }^{1}$ Bronchial asthma is characterized by paroxysmal dyspnoea accompanied by wheezing resulting from narrowing bronchial airways by muscle spasms, mucosal swelling, or viscous secretion. As per Ayurveda, Shwasa is mainly caused by Vata and Kapha doshas. There are five types of Shwasaroga, Tamak Shwasa is one of the types of Shwasa roga, it is mentioned as Yaapya Vyadhi. ${ }^{2}$

\section{NIRUKTI OF SHWASA ROGA: 'Shwasitam Vayu iti Shwasah. ${ }^{3}$ \\ Inhalation and exhalation of air mean Shwasa.}

'ShwasatiAnenaiti Shwasah ${ }^{4}$ - The process of the exchange of air is Shwasa. Acharya Charaka explains that Tamaka Shwasa gets aggravated when exposed to a cloudy atmosphere, cold water, cold weather, and wind blowing from the eastern direction. In Tamak Shwasa, the direction of Udana Vayu changes, i.e. the normal Anulomanagati of Udana Vayu becomes Pratiloma, Sirasa get obstructed because of this patient get difficulty in respiration.

NIDANA /HETU(Etiology): Vata Vardhak Aahara-Vihara may cause Dhatu Kshaya and Vata Propoka. ${ }^{5}$ Kapha Vardhak Aahara-Vihara causes Agnimandhya result in production of Ama. The Ama having similar Gunaas Kapha causes Kapha Prakopa.
SAMPRAPTI (Pathogenesis) ${ }^{6}$ : In Shwasa udana yavu get obstructed, i.e. the normal Anulomanagati of Udana Vayu becomes Pratiloma, Sirasa get blocked because of this. Then patients have difficulty in respiration, i.e. dyspnoea.

Apathya Vihara as exposure to the raja, dhuma, aghata, etc.

Khavaigunya

Kapha vikruti in Pranavaha srotas

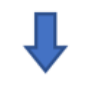

Sroto vikruti= Sanga, Vimarggaman

Obstruction to the normal flow of Prana vayu

Vimarga/ pratilomagamana of prana vayu

Shwasa urdhwagamanam

Tamaka Shwasa 


\section{SampraptI Ghataka}

\begin{tabular}{|l|l|}
\hline Hetu: Vata kapha vardhaka Aahara Vihara & Adhistana: Uras \\
\hline Dosha: Vata-Kapha & Sanchar Sthana: Ura kantha \\
\hline Dushya: Rasa Dhatu & Vyakt Sthana: Urasa \\
\hline Agni: Jatharagni, Rasadhatvagni & Rogamarga: Aabhyantara \\
\hline Strotas: Prana-Udaka-Annavaha strotasa & Srotodushtiprakar- a) Sanga b) Vimarggramana \\
\hline Udbhav Shtana: Pitta Sthana (Amashaya) & Sadhyasadhatva: Yapya \\
\hline
\end{tabular}

Purva Roopa (Prodromal symptoms of Shwasa) ${ }^{7}$ : Anaha, Adhmana, Arati, Bhaktdwesha, Hritpeeda, Kantha gurutwa, Parshwa shola, Prana vilomata, Shankhatoda and Uroguruta.

Roopa (Symptoms) ${ }^{7}$ : Ghurghurkam (Wheezing), Shwasa krichrata (Dyspnoea), Shayanasysa Shwasapeedita (Dyspnoea increases in lying position), Peenas (Coryza), Kasa (Cough), Kantodhwansha (Throat irritation), Lalat Sweda (Sweating in the forehead), Ateevteevravega shwasa (increase rate of respiration), Prana prapeedana (Tightness in the chest).

Sadhya-Asadhyata: According to Charaka, it is yapyavyadhi, i.e., difficult to cure. If it is in Navavastha then it is Sadhya. ${ }^{8}$ Tamaka Shwasa is curable in a strong person when the Shwasa symptoms are not fully manifested ${ }^{9}$ According to Vagbhata, it is Yapya, but if it is treated in the beginning and the patient is strong (balina), then Shwasa is Sadhya ${ }^{10}$.

\section{Observation}

Most of the ingredients of pushkarmooladi yoga show a pharmacological property of Ushna Virya, Katu-Tikta rasa, Laghu Guna and Vata kapha har property. All the ingredients of
Pushkarmooladi yoga have the quality to normalize prakupita Vatakapha dosha. The drug Pushkarmooladi yoga produce a significant relaxation of bronchioles. Shwasa roga is caused by the sang and vimarggaman of strotas and kapha prakopa, Ushna Virya, Katu, Tikta rasa, and Shwasa hara property of ingredients act at this instance. The Pusharmooladi prove an effective medicine for treatment to reduce Vata kapha predominant Shwasa.

Ingredients of Pusharmooadi yoga shows antimicrobial activity against certain gram + ve and gram-ve bacteria, which are causing recurrent respiratory infection, which is responsible for predisposing factor Shwasa roga. Pushramooladi yoga offers excellent antimicrobial activity against many bacteria causing RTI and helps in reducing the severity of Shwasa.

The Phytochemical study shows that Pushkarmooladi yoga has many Phytochemical-like phenolic compounds and natural steroids, which prevent the release of free radicals and act as an antioxidant, in this way helps in reducing the severity of Shwasa. It also shows bronchodilator and expectorant property. By these property Pushkarmooladi yoga help in breaking the pathogenesis of Shwasa roga.

Table 1: Pharmacological Property of Pushkarmoola (Inula racemosa)

\begin{tabular}{|c|c|c|c|c|c|}
\hline Rasa & Guna & Virya & Vipaka & Dosik action & Use/Action \\
\hline Katu, TiktaII & Laghu & Ushna & Katu & Kapha hara & Shwasa, kasa, Hikka, Parshwashoola, Aruchi \\
\hline Katu, Tikta & Snigdha & Ushna & Katu & Vata Kapha Shamaka & Shwasa, Kasa, Hikka, Parshwashoola, Aruchi, Jwara \\
\hline Tikta, Katu $^{\text {I3 }}$ & - & Ushna & - & Vata Kapha hara & Shwasa, Kasa, Hikka, Shotha, Adhmana \\
\hline
\end{tabular}

Table 2: Pharmacological Property of Pippali (Piper longum)

\begin{tabular}{|c|c|c|c|c|c|}
\hline Rasa & Guna & Virya & Vipaka & Dosik action & Use/Action \\
\hline Katu $^{14}$ & - & Anushna Shita & Madhura & Vata, Kapha Shamaka & $\begin{array}{c}\text { Amatisar (Charak Samhita chikitsa, } \\
\text { Deepan, Pachana. }\end{array}$ \\
\hline Katu $^{15}$ & Laghu, Snigha & Anushna, Shita & Madhura & Vata, Kapha Shamaka & Shwasa, Kasa. \\
\hline Katu $^{16}$ & Tikshna & Ushna & Madhura & Vata, Kapha Shamaka & $\begin{array}{c}\text { Shwasa, Kasa, Deepana, Pachana and } \\
\text { Rasayana }\end{array}$ \\
\hline Katu $^{17}$ & Snigdha & Ushna & Madhura & Vata, Kapha Shamaka & Shwasa, kasa, Deepana \\
\hline
\end{tabular}

Table 3: Pharmacological Property of Dhanvayasa (Fagonia cretica)

\begin{tabular}{|c|c|c|c|c|c|}
\hline Rasa & Guna & Virya & Vipaka & Dosik action & Use/ Action \\
\hline Tikta $^{18}$ & - & Shita & - & Kapha hara & Jwara, Mutrajanan, Vranropak \\
\hline${\text { Tikta, } \text { Kashaya }^{19}}^{2}$ & Laghu & Shita & Madhura & Kapha Pitta hara & Kasa, Jwara, Vaman, Medohar \\
\hline Tikta $^{20}$ & - & Shita & Madhura & - & Dahavinashak, Visham jwara, Chardi, Mohvinashka \\
\hline
\end{tabular}

Table 4: Pharmacological Property of Karkatshringi (Pistacia integerrima)

\begin{tabular}{|c|c|c|c|c|c|}
\hline Rasa & Guna & Virya & Vipaka & Dosik action & Use/ Action \\
\hline Kashaya, Tikta ${ }^{21}$ & Ruksha & Ushna & Katu & Kapha Vata Shamaka & Shwasa, Kasa, Hikka, Jwara \\
\hline Kashaya, Tikta ${ }^{22}$ & Laghu & Ushna & Katu & Kapha Vata Shamaka & Shwasa, Kasa, Jwara, Hikka \\
\hline All rasa except Lavana ${ }^{23}$ & Laghu & - & - & Tridosha Shamaka & Kasa \\
\hline
\end{tabular}

Table 5: Pharmacological Property of Ativisha (Aconitum heterophyllum)

\begin{tabular}{|c|c|c|c|c|c|}
\hline Rasa & Guna & Virya & Vipaka & Dosik action & Use/ Action \\
\hline${\text { Katu, } \text { Tikta }^{24}}^{24}$ & - & Ushna & Katu & Kapha Pitta Shamaka & Kasa, Jwara, Aamvisha, Krimi \\
\hline Tikta $^{25}$ & Laghu & Ushna & Katu & Kapha Pitta Shamaka & Kasa, Jwara, Aamvisha, Krimi \\
\hline Katu $^{26}$, Tikta & Laghu & Ushna & Katu & Kapha Pitta Shamaka & Kasa, Jwara, Aamvisha, Krimi \\
\hline
\end{tabular}


Table 6: Antimicrobial Activity of Pushkarmoola (Inula racemosa)

\begin{tabular}{|c|c|c|}
\hline Name of Bacteria & In Vivo or in Vitro & Result \\
\hline S. aureus, E. coli. ${ }^{27}$ & In vitro & $\begin{array}{l}\text { Inula racemose has antimicrobial properties against } \\
\text { listed bacteria }\end{array}$ \\
\hline $\begin{array}{c}\text { Gram. +ve, gram -ve } \\
\text { Staphylococcus aureus, Serratia Marcescens, E. coli, } \\
\text { Shigella dysentariae, S. typhi, p. aeruginosa }{ }^{28}\end{array}$ & In vitro & $\begin{array}{c}\text { The aqueous extract of this plant seed has } \\
\text { antimicrobial activity. }\end{array}$ \\
\hline S. aureus, E. coli, S. aureus ${ }^{29}$ & In vitro & $\begin{array}{c}\text { Inula racemosa has antibacterial properties against } \\
\text { both gram +ve and gram. -ve }\end{array}$ \\
\hline
\end{tabular}

Table 7: Antimicrobial Activity of Pippali (Piper longum)

\begin{tabular}{|c|c|c|}
\hline Name of Bacteria & In Vivo or in Vitro & Result \\
\hline $\begin{array}{c}\text { S. alba, E. coli, B. megaterium, Aspergillus } \\
\text { aeruginosa } 30\end{array}$ & In vitro & $\begin{array}{l}\text { In vitro study proves antibacterial property of piper } \\
\text { longum }\end{array}$ \\
\hline S. pyogenes, K. pneumoniae, S. aureus ${ }^{31}$ & In vitro & $\begin{array}{l}\text { Piper longum has antimicrobial properties against } E \text {. } \\
\text { histolytica }\end{array}$ \\
\hline $\begin{array}{c}\text { K. pneumoniae, Pseudomonas aeruginosa, } \\
\text { Staphylococcus aureus. }{ }^{32}\end{array}$ & In vitro & The plant is effective against the listed bacteria. \\
\hline
\end{tabular}

Table 8: Antimicrobial Activity of Karkatshringi (Pistacia integerrima)

\begin{tabular}{|c|c|c|}
\hline Name of Bacteria & In Vivo or in Vitro & Result \\
\hline $\begin{array}{c}\text { Staphylococcus aureus, E. coli, Pseudomonas } \\
\text { aeruginosa }\end{array}$ & In Vitro & $\begin{array}{l}\text { Pistacia integerrima shows antimicrobial activity in } \\
\text { different concentrations. }\end{array}$ \\
\hline $\begin{array}{c}\text { Staphylococcus aureus, Salmonella typhi, B. subtilis, } \\
\text { Pseudomonas, aeruginosa, E. faecalis }{ }^{34}\end{array}$ & In Vitro & $\begin{array}{c}\text { Pistacia integerrima shows antimicrobial activity in } \\
\text { different concentrations. }\end{array}$ \\
\hline B. subtilis, S. aureus, Pseudomonas pickettii ${ }^{35}$ & In Vitro & $\begin{array}{l}\text { Ethyl acetate fraction of Pistacia integerrima shows } \\
\text { antimicrobial inhibition against bacteria. }\end{array}$ \\
\hline
\end{tabular}

Table 9: Antimicrobial Activity of Dhanvyasa (Fagonia cretica)

\begin{tabular}{|c|c|c|}
\hline Name of Bacteria & In Vivo or in Vitro & Result \\
\hline $\begin{array}{c}\text { Gram +ve, gram -ve, E. coli, Staphylococcus aureus, } \\
\text { Streptococcus pyogenes and fungal stain Candida } \\
\text { albicans }\end{array}$ & In vitro & $\begin{array}{l}\text { The Gram +ve bacteria \& gram -ve bacteria both are } \\
\text { susceptible. }\end{array}$ \\
\hline $\begin{array}{c}\text { S. aureus, E. coli, K. pneumonia, Enterobacter } \\
\text { aerogenes, Bordetella Bronchiseptica }{ }^{37}\end{array}$ & In vitro & Antimicrobial action against listed bacteria. \\
\hline $\begin{array}{c}\text { Gram +ve, Gram -ve, E. coli, S. aureus } \\
\text { Staphylococcus epidermidis } \\
\text { Pseudomonas aeruginosa, Bacillus Subtilis }{ }^{38}\end{array}$ & In Vitro & $\begin{array}{l}\text { The Gram +ve bacteria \& gram-ve bacteria both are } \\
\text { susceptible. }\end{array}$ \\
\hline
\end{tabular}

Table 10: Antimicrobial Activity of Ativisha (Aconitum heterophyllum)

\begin{tabular}{|c|c|c|}
\hline Name of Bacteria & In Vivo or in Vitro & Result \\
\hline $\begin{array}{l}\text { Streptococcus aureus, } B . \text { bronchiseptica, B. subtilis, } P . \\
\text { putida and } X \text {. campestris antimicrobial activity } \\
{ }^{39}\end{array}$ & In vitro & Aconitum hererophyllum shows antibacterial activity \\
\hline $\begin{array}{l}\text { B. cereus, } S \text {. aureus, Pseudomonas aeruginosa, Salmonella } \\
\text { typhi, Serratia marcescens, E. coli, K. pneumonia }{ }^{40}\end{array}$ & In vitro & $\begin{array}{l}\text { Aconitum hererophyllum has antibacterial properties } \\
\text { against both grams. + ve and gram-ve bacteria }\end{array}$ \\
\hline $\begin{array}{c}\text { Staphylococcus aureus, Bacillus subtilis, Candida albicans } \\
\text { and Aspergillus flavus }{ }^{41}\end{array}$ & In vitro & $\begin{array}{l}\text { Aconitum hererophyllum is having antibacterial } \\
\text { against listed bacteria }\end{array}$ \\
\hline
\end{tabular}

Table 11: Phytochemical Studies of Ingredients of Pushkarmoola yoga

\begin{tabular}{|c|c|}
\hline Drugs Name & Phytochemical constituents \\
\hline $\begin{array}{l}\text { Pushkarmoola } \\
\text { (Inula racemosa) }^{42}\end{array}$ & $\begin{array}{c}\text { Alantolactone, Isoalatolactone, Inunolide, Dihydroisoalanto lactone, B- sitosterol, D-mannitol, } \\
\text { Dihydroxinunolide, Neoalantolactone, in-uronile }\end{array}$ \\
\hline Pippali (Piper longum) $)^{43}$ & Piperine, Tannin, Piplartine, Pipernonaline, n-hexaadecane, n-heptadecane \\
\hline $\begin{array}{c}\text { Karkatshring } \\
(\text { Pistacia integerrima) } \\
\text { (44 }\end{array}$ & $\begin{array}{l}\text { Tannin, Resin, Essential oil, Pistacienoic acid, Pistacin oil acid B, B- sitosterol and } \\
\text { Masticadienoic acid }\end{array}$ \\
\hline $\begin{array}{c}\text { Dhanvyasa } \\
(\text { Fagonia cretica })^{45}\end{array}$ & Tannin, Saponin, Steroid, Alkaloids, and Glycosides \\
\hline $\begin{array}{c}\text { Ativisha } \\
\text { (Aconitum heterophyllum) }^{46}\end{array}$ & $\begin{array}{c}\text { Atisine, Atidine, Tanic acid, Aconitic acid, B-sitosterol, Heterophyllcene, Heterophylline, } \\
\text { Isoatisine }\end{array}$ \\
\hline
\end{tabular}

\section{CONCLUSION}

The pharmacological properties of drugs help break down the pathogenesis of Shwasa roga. Every ingredient shows antibacterial property up to some extent against certain Gram +ve and Gram-ve bacteria causing recurrent respiratory tract infection and is responsible for Shwasa. The phytochemical property offers that pushakarmooladi yoga causes certain phenolic compounds and natural steroid, acting as an antioxidant, anti-inflammatory and bronchodilator. Based on the pharmacological property, antimicrobial study, phytochemical study, bronchodilator and expectorant property, it is concluded as Puskarmooladi yoga seem to be an effective and safe formation of treatment Shwasa raga. 


\section{REFERENCES}

1. Dharmage SC, Perret JL, Custovic A. Epidemiology of Asthma in Children and Adults. Front Pediatr. 2019; 7:246. Published 2019 Jun 18. doi:10.3389/fped.2019.00246

2. Acharya Trikamji Yadavji, Charaka Samhita of Agnivesha, Chakrapani Datta, Ayurveda Deepika (Sans) Chikitsa, 17/5556 Vol-5, Varanasi Chaukhambha Surbharati Prakashan, Verse no.20, reprint2011. P 535

3. Raja Radhakantha decompiled Shabdakal padhruma, Vol-5, Publisher by Naga Publisher, New Delhi 1987, Verse No- 26, $555 \mathrm{P}, 177$.

4. Singh Kushawaha Harish Chandra (ed). Charka Samhita. Chikitsa sthana, Shwasa roga chikitsa Reprint ed.2012, Vol II Varanasi; Chaukhamba orientalis Varanasi 2012, verse no.45, 1172 P 456.

5. Acharya Trikamji Yadavji, Charaka Samhita of Agnivesha, Chakrapani Datta, Ayurveda Deepika (Sans) Chikitsa 17, reprint Edition 2011, Vol-5, Chaukhambha Surbharati Prakashan, Verse no. 25, P. 533.

6. Acharya Trikamji Yadavji, Charaka Samhita of Agnivesha, Chakrapani Datta, Ayurveda Deepika (Sans) Chikitsa, 17/5556 Vol-5, reprint edition 2011 Varanasi Chaukhambha Surbharati Prakashan, P. 533.

7. Acharya Trikamji Yadavji, Charaka Samhita of Agnivesha, Chakrapani Datta, Ayurveda Deepika (Sans) Chikitsa17, Varanasi Chaukhambha Surbharati Prakashan, reprint 2011. P 535.

8. Singh Kushawaha Harish Chandra (ed), Charaka Samhita 55, Shwasa chikitsa 17/12 of Agnivesha, reprint ed., vol II, Verse no. 30 Varanasi; Chaukhamba Orientalia; 2012, 1172 chikitsa 17/12. P. 454-55

9. Singh Kushawaha Harish Chandra (ed), Charaka Samhita 55, Shwasa chikitsa 17/12 of Agnivesha, reprint ed., Vol II Varanasi; Chaukhamba Orientalia; 2012, 1172 P 454.

10. Tripathi Brahmanand (ed), Ashtanga Hridaya Nidana sthana Swasahi dhmaadhyaya $4 / 10$ of Shimadh vaghata, Vol-1, Verse: 5, 2011Chaukhamba Sanskrit Pratikshan Delhi2011. P. 455.

11. Krishna Chandra Chunekar and Dr.Gangasahaya, Bhavprakasha, Harityakadi Varga 57, Chaukhambha Bharti academy, Varanasi-221001.1999.

12. Priya vrata Sharma and Dr.Guruprasad Sharma KaiyadevaNighantuh, Oshadhi Varga 57, edited and translated, Chaukhamba Prakashna Varanasi, reprint 2008.

13. Priya Vrat Sharma and Dr. Guru Prasad Sharma Dhanvantari nighntu, Chaukhamba Orientalia, Varanasi, 1981.

14. Pandit Kashnath Shastri and Dr.Gorkhnath Chaturvedi, Charka Samhita 'Vidotini' Hindi Commentary, Vol. 2, Chaukhambha Bhartiya academy Varanasi-221001 (India).

15. Krishna Chandra Chunekar and Dr. Gangasahaya Pandey, Bhavprakash Nighantuh, Harityakadi Varga 57, Chaukhambha Bharti academy, Varanasi, 2013.

16. Priya vrata Sharma and Dr. Guruprasad Sharma KaiyadevaNighantuh, Oshadhi Varga, edited and translated, Chaukhamba Prakashna Varanasi, reprint 2008.

17. Priya Vrat Sharma and Dr. Guru Prasad Sharma Dhanvantari nighntu, Vol.1 Chaukhamba Orientalia, Varanasi, 1998.

18. Krishna Chandra Chunekar and Dr.Gangasahaya Pandey, HarityakadiVarga57,Bhavprakash Nighantuh, Haritakyadi Varga 112 by Chaukhmbha Bharti academy, Varanasi.1999.

19. Priya vrata Sharma and Dr. Guruprasad Sharma, KaiyadevaNighantuh, Oshadhi Varga 13-14, Chaukhamba Prakashan Varanasi, reprint 2008 .

20. Priya Vrat Sharma and Dr. Guru Prasad Sharma Dhanvantari nighntu 7, Chaukhamba Orientalia Varanasi, 1998.

21. Krishna Chandra Chunekar and Dr.Gangasahaya Pandey, Bhavprakash Nighantuh, Haritakyadi Varga 112,
Harityakadi Varga 57, Chaukhambha Bharti academy, Varanasi-221001. 1999.

22. Priya vrata Sharma and Dr. Guruprasad Sharma KaiyadevaNighantuh, Oshadhi Varga 13-14, Chaukhmba Prakashna Varanasi, reprint 2008.

23. Priya Vrat Sharma and Dr. Guru Prasad Sharma, Dhanvantari nighntu 19, Chaukhamba Orientalia, Varanasi,1998.

24. Krishna Chandra Chunekar and Dr.Gangasahaya Pandey Bhavprakash Nighantuh, Guduchyadi Varga 89-90 Varga 112, Harityakadi Varga 57, Chaukhambha Bharti academy, Varanasi-221001. 1999.

25. Priya vrata Sharma and Dr. Guruprasad Sharma KaiyadevaNighantuh, Oshadhi Varga 13-14,Chaukhmba Prakashna Varanasi, reprint 2008.

26. Priya Vrat Sharma and Dr. Guru Prasad Sharma, Dhanvantari nighntu, edited by Chaukhamba Orientalia, Varanasi, 1998.

27. Mangesh Krashna, Rohit Sarswat, Antimicrobial Activity of Inula racemose, International Journal of Chemical and Pharmaceutical Analysis, 2020; 3 (4): 1-9.

28. P.D. Lokhande, K.R. Gawai, K.M. Kodam, B.S. Kuchekar, Complete Review of Inula racemose, Research Journal of Medicinal Plant. 2007; 1: 7-12.

29. Alper Gökbulut, Onural Özhan, Basri Satılmış, Kadir Batçığlu, Selami Günal, Engin Şarer, Antioxidant and Antimicrobial Activities, and Phenolic Compounds of Selected Inula species from Turkey, Natural Product Communications 2013; 8(4): 475 - 478.

30. Mohib Khan and Mustafa Siddiqui, Complete Review on Piper longum, Journal of Natural Product Radiance, 2007; 6 (2): 111-113.

31. Aparna Saraf, Amit Saraf, Extraction of Phytochemicals and Antimicrobial activity of Piper longum, International Journal of Pharmacognosy and Phytochemical Research 2014; 6 (4): 213-222.

32. Chandan Singh, Santosh K. Singh, Gopal Nath and N.P. Rai, Anti-mycobacterial activity of Piper longum L. fruit extracts against multi drug-resistant mycobacterium spp, International Journal of Phytomedicine. 2011; 2 (3): 353-361.

33. Pramod D Khobragade, Minal Khobragade, Digambar S Chothe, Phytochemical analysis and antimicrobial activity of galls of Pistacia integerrima stew ex. Brand, International Journal of Ayurvedic Medicine 2014; 3 (5): 76-81.

34. Ghias Uddin and Abdur Rauf, Antimicrobial activity of Pistacia integerrima gall, Middle-East Journal of Medicinal Plants Research. 2012; 1(2): 36-40.

35. Y L Ramchandra, B.E. Ravi Shankara, P.S. Sujan Ganopathy, S. Sundar Rajan, Phytochemicals and Antimicrobial activity, An International Research Journal of Pharmacophore. 2010; 1 (2): 149-154.

36. Amatul basit, Rashida sultana and Abida Aziz, university of Lahore, Nusrat Jahan College Rabwah, Extraction of phytochemicals from Fagonia arabica and their antimicrobial activity, International Journal of Chemical and Biochemical science, 2019; 15:25-27

37. Rumana Saeed, Rashid Iqubal, Hameed Ur Rahman, Nisar Ahm ad, Zubia Masood, Antibacterial and Phytochemical Evaluation of the crude extract and Fractions of Fagonia cretica, International Journal of Pharma Science and Research. 2015; 6 (2): 278-281.

38. Sajid B, Alia E, Rizwana K, Uzma S, Alameer, Hafiz M I, Antimicrobial activity of plant Fagonia cretica, Journal of Pharmacy Research. 2011; 4(4): 962-963.

39. Nidhi Srivastava, Vikas Sharma, Kriti Saraf, Anup Kumar Dobriyal Indian, A Complete Review on Ativisha- Aconitum heterophyllum, Journal of Natural Products and Resources. 2011; 2 (4): 504-507.

40. Tejas Joshi and Sunetra Chaudhari, Phytochemical \& antimicrobial activity of Aconitum heterophyllum, 
International Journal of Scientific Research and Reviews. 2019;8(3):323-330.

41. Rashi Jaiswal, Dr. Jyoti Kaushik and Prof. Rakesh Sharma, Phytochemical and Antimicrobial Activity of Aconitum heterophyllum, World Journal of Pharmaceutical and Medical Research, 2020; 6(7):132-135.

42. Manish Kumar Soni and Dr. Omprakash Sharma, Phytochemical Screening of Inula racemosa World journal of pharmaceutical and medical research, 2018; 4 (3): 199-201.

43. Chauhan Khushbu et al, Phytochemical \& Antimicrobial activity of Piper longum, International Journals of Research in Ayurveda \& Pharmacy 2011; 2(3): 157-160.

44. Promod D Khobragade, Minal Khobragade, Digambar S Chothe, Phytochemical and Pharmacological property of Pistacia integerrima, International journal of Ayurvedic medicine, 2014; 5(1): 76-81.
45. Khuswinder Kaur, Sandeep Bain and Summet Grewal, International Journal of Ayurvedic Medicine 2014; 4 (4): 962-963.

46. Gurudeeban, S., Rajamanickam,E., Ramanathan, T and Satyavani, K .Antimicrobial Activity of Citrullus colocynthis In Gulf Of Mannar. International Journal of Current Research , 2010; 2: 078-081.

\section{Cite this article as:}

Khobragade Swapnil and Upadhyay Prem Shanker. A critical review on pushkarmooladi yoga in shwasa roga. Int. J. Res. Ayurveda Pharm. 2021;12(6):64-68 http://dx.doi.org/10.7897/ 2277-4343.1206172

\section{Source of support: Nil, Conflict of interest: None Declared}

Disclaimer: IJRAP is solely owned by Moksha Publishing House - A non-profit publishing house, dedicated to publishing quality research, while every effort has been taken to verify the accuracy of the content published in our Journal. IJRAP cannot accept any responsibility or liability for the site content and articles published. The views expressed in articles by our contributing authors are not necessarily those of IJRAP editor or editorial board members. 